\title{
Motor unit potential abnormalities in multiple sclerosis: further evidence for a peripheral nervous system defect
}

\author{
ANDREW I WEIR, STIG HANSEN, AND JOHN P BALLANTYNE \\ From the Glasgow University Department of Neurology and Department of Clinical Physics and \\ Bio-engineering Institute of Neurological Sciences, Southern General Hospital, Glasgow
}

SUMMARY We have recently reported abnormalities of single fibre EMG in patients with multiple sclerosis. The present study applies quantitative electrophysiological techniques to the same group of patients. The number of motor units in the extensor digitorum brevis muscle was measured and their characteristics recorded. Also the shortest distal motor latency and fastest motor conduction velocities were estimated. Abnormalities suggesting a patchy denervating/reinnervating process due to pathology in the intramuscular nerve network or at the endplate were found in a number of patients. There was a good correlation between patients with abnormal motor unit potentials and those with abnormal single fibre EMG "jitter."

The traditional concept that mutiple sclerosis is a disease confined to the central nervous system (CNS) has been challenged on pathological ${ }^{1}$ and electrophysiological grounds. ${ }^{2}{ }^{3}$ We have recently reported results of a single fibre electromyographic study of patients with this disease showing significant abnormalities in the peripheral nervous system (PNS). ${ }^{4}$ The present study of the same group of patients involves estimation of the motor unit numbers and motor unit potential parameters as a further sensitive test of peripheral nerve and muscle function.

The infrequent reports of neuropathological investigations of the PNS in multiple sclerosis have already been reviewed. ${ }^{4}$ In summary there were three main postmortem findings:

(a) no abnormality, ${ }^{5}$

(b) significant distal demyelination in the peripheral nerves of emaciated patients thought to be due to a nutritional neuropathy, ${ }^{6}$

(c) hypertrophic neuropathy in a few single case reports, ${ }^{7-9}$ and a review of four cases where the neuropathy predominated proximally in the spinal roots. ${ }^{10}$

In addition, Pollock et al have undertaken sural nerve biopsies in patients with undoubted multiple sclerosis but with minimal disability and have shown a significant percentage of patients with abnormal internodes characterised by reduced

Address for reprint requests: Dr AI Weir, Institute of Neurological Sciences, Southern General Hospital, Glasgow G51 4TF, Scotland.

Accepted 28 May 1980 myelin thickness due to a loss of myelin lamellae without change in the axonal cross-sectional area.

Electrophysiological studies of peripheral nerve conduction in multiple sclerosis have been normal using conventional techniques. ${ }^{11-13}$ However, Hopf has shown reduced conduction velocities in small diameter motor nerve fibres ${ }^{2}$ and an increase in relative refractory periods in median nerve sensory fibres. ${ }^{3}$ These results suggest that there is a PNS defect in this disease but detectable only by sensitive techniques. Our recent study of the single fibre electromyographic jitter (SFEMG jitter) showed that of a group of 15 patients with "definite" multiple sclerosis, ${ }^{14}$ two had a previously undiagnosed peripheral neuropathy. The latter was however detected clinically and confirmed electrophysiologically at the time of this investigation. A further four patients had clearly abnormal jitter. Of the remaining nine patients, five had minor abnormalities whilst four were entirely normal. Ten of the 15 patients had a mean jitter above the accepted upper limit of normal. ${ }^{15}$

\section{Subjects and methods}

The 15 patients have been supplemented by a further seven for the present investigation. The criteria for inclusion were similar: (a) all had been investigated in the Institute of Neurological Sciences, Glasgow within the past four years, (b) all fulfilled the criteria of "definite" multiple sclerosis (Schumacher 14) (c) all had confirmatory investigations including abnormal $\mathrm{IgG} /$ total protein ratio in the CSF and/or prolonged 
or abnormal visual evoked potentials. Drug histories of the patients showed no known neurotoxic preparations although most had received a course of corticotrophin at diagnosis but none in the year prior to this study. Informed consent was obtained from the patients prior to examination. The investigations were carried out in a thermostatically controlled room and the patients' skin temperature over the muscle under investigation was maintained at $33 \pm$ $1{ }^{\circ} \mathrm{C}$.

The methods of recording and analysing the motor unit potentials in the extensor digitorum brevis (EDB) muscle have been described in detail in previous papers. ${ }^{16} 17$ In summary, motor unit potentials (MUPs), recorded from surface electrodes over the EDB muscle are evoked sequentially by finely graded incremental stimulation of the anterior tibial nerve at the ankle. Recruitment of up to 15 motor units can be recognised by a combination of visual and computer analysis of muscle action potential increments displayed on the oscilloscope screen. The first the potential of the second is incorporated in a compound muscle action potential containing MUPs 1 and 2. As each new potential is added to the preceding, one compound muscle action potential is stored in a computer memory (Template). Template 1 contains MUP 1 , template 2 contains the sum of MUPs 1 and 2; template 3 contains the sum of MUPs 1, 2 and 3 and so on. Up to 15 templates can be stored. The number of motor units in the EDB muscle is calculated from the formula

$$
\mathrm{MUC}=\frac{\mathrm{A}(\mathrm{M})}{\mathrm{A}(\mathrm{N})} \times \mathrm{N}
$$

where $A(M)$ is the area of the supramaximally evoked muscle action potential and $A(N)$ is the area of the compound muscle action potential containing N MUPs.

By a process of template subtraction, the computer also displays the first and sequentially recruited MUPs in isolation, for example, subtraction of template 1 from template 2 will leave MUP 2 in isolation; subtraction of template 2 from template 3 will leave MUP 3 in isolation and so on. The latencies, durations, areas and amplitudes of individual MUP is displayed in isolation on the oscilloscope;

MUPs are then measured either by the computer or by hand on a printout. The fastest motor nerve conduction velocities (FMNCV) were obtained by conventional surface recording techniques ${ }^{18}$ of the evoked potential in the EDB muscle using the same undisturbed electrodes as the motor unit potential study. The anterior tibial nerve was supramaximally stimulated at the ankle and the common peroneal nerve at the head of the fibula. In all the patients the conduction velocity measurements were performed on the right common peroneal nerve and the MUP estimations on the right EDB muscle whatever the location of the patient's symptoms or signs. All patients were examined clinically for signs of a peripheral neuropathy. Three of the 22 patients had overt clinical evidence of a peripheral neuropathy but are included in the results section as a subgroup for comparison. In none of these patients was an obvious cause for neuropathy found. The 27 control subjects aged $46.9 \pm 12.4$ years were members or relatives of the staff of the Institute of Neurological sciences, Glasgow and had no evidence of neurological disease.

\section{Results}

The results shown in Tables 1 to 3 are expresse as the mean \pm 1 standard deviation and the statistical significance estimated using Students $t$ test. In the tables the patients have been divB ded into subgroups as follows (see table 1).

$\begin{array}{ll}\text { Line } \mathrm{a}= & \text { control values. } \\ \text { Line } \mathrm{b}= & \begin{array}{l}\text { All the patients with multi- } \\ \text { ple sclerosis. }\end{array}= \\ \text { Line } \mathrm{c}= & \begin{array}{l}\text { Patients with a clinical per- } \\ \text { ipheral neuropathy. }\end{array} \\ = & \begin{array}{l}\text { Patients without a clinical } \\ \text { neuropathy. }\end{array} \\ \text { Line } \mathrm{d} & \begin{array}{l}\text { Subdivision of patients with- } \\ \text { out neuropathy by abnormal } \\ \text { or normal SFEMG jitter. }\end{array} \\ \text { (Therefore } \mathrm{b}= & \mathrm{c}+\mathrm{d} \text { and } \mathrm{d}=\mathrm{e}+\mathrm{f}) .\end{array}$

Table 1 Age and duration of symptoms

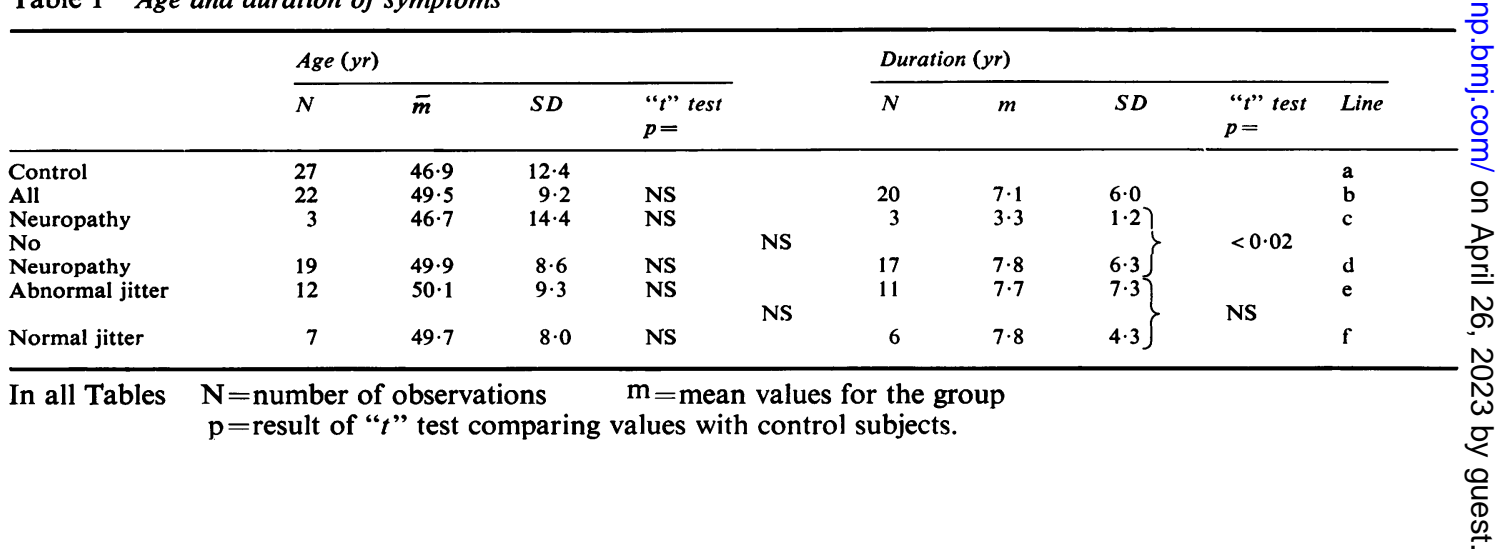


Table 2 Shortest distal motor latency, fastest motor conduction velocity and the area under the supramaximal compound action potential

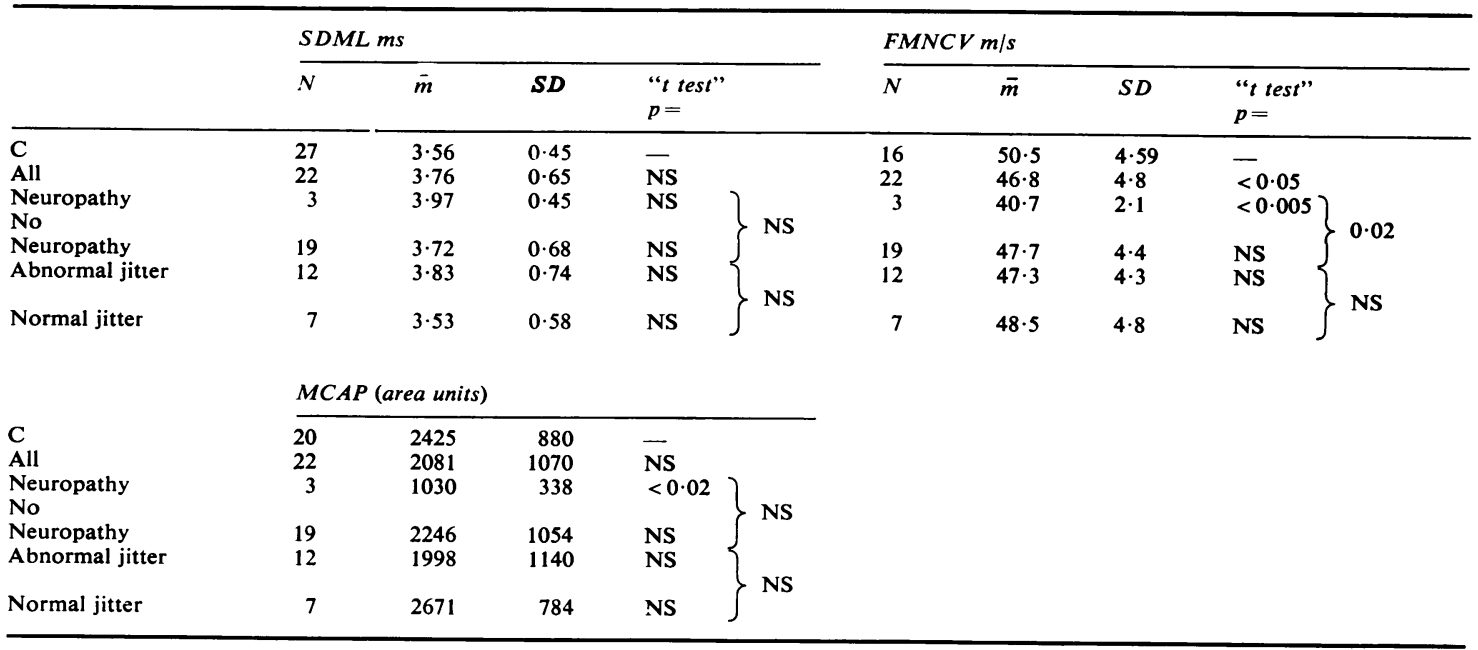

Table 3 Motor unit parameters

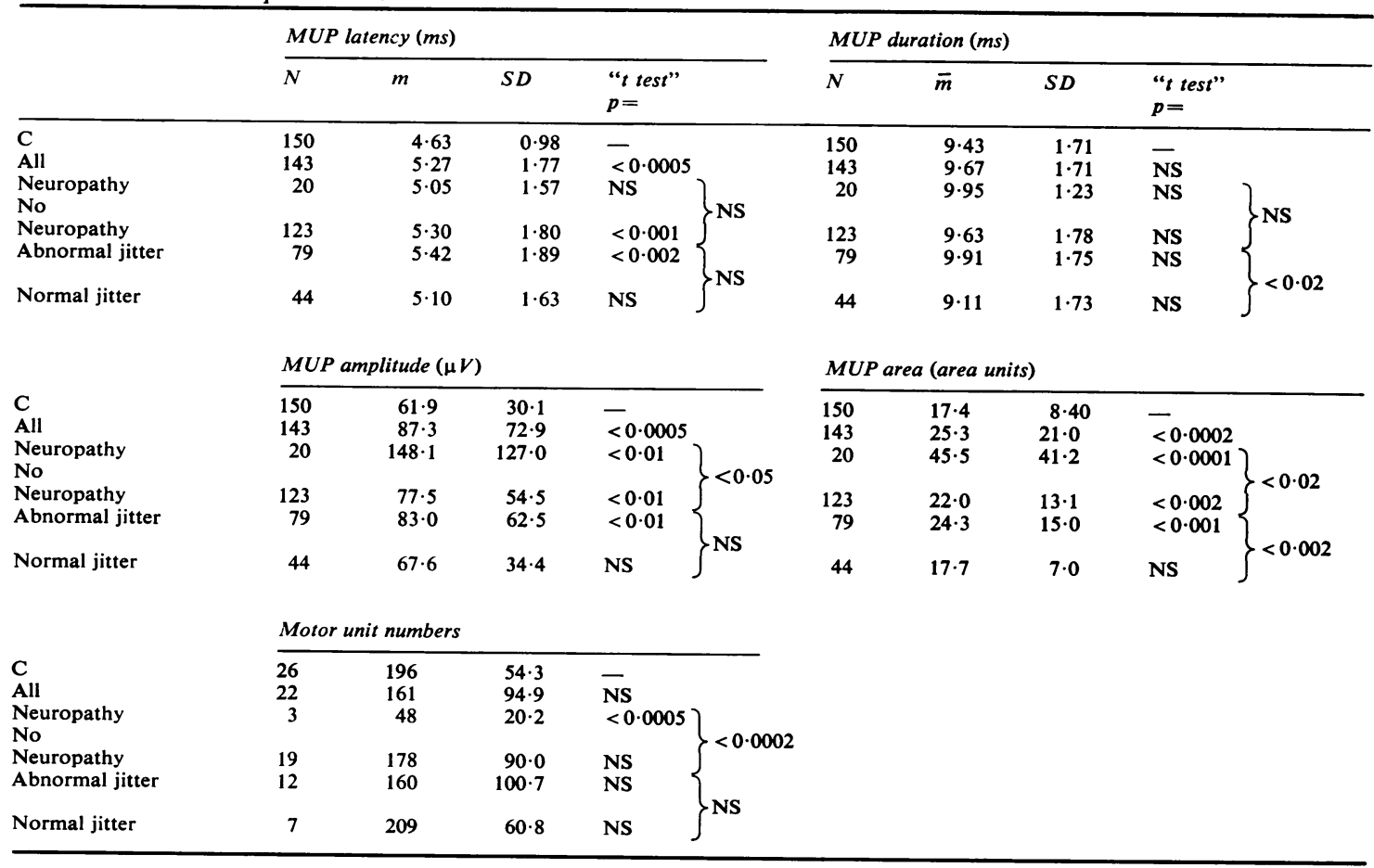

The definitions of SFEMG abnormality have been detailed elsewhere, ${ }^{4151920}$ but in summary our criteria of abnormality were (a) two potential pairs with a mean consecutive difference (MCD) greater than $55 \mu \mathrm{s}$; or (b) one of six potential pairs with an MCD greater than $55 \mu \mathrm{s}$ plus a mean MCD greater than $34 \mu \mathrm{s}$. Each group was compared statistically with the control group and a further test of significance was made between the groups with and without clinical 
neuropathy and with and without abnormal SFEMG jitter.

Table 1 indicates the mean age and duration of symptoms in each group. The three patients with a clinical neuropathy had a significantly shorter duration of illness than the others. However, as the numbers with neuropathy are small no great weight can be placed on this result. Table 2 shows the mean shortest distal motor latency (SDML), mean fastest motor conduction velocity (FMNCV) and the mean area of the supramaximal compound action potential (MCAP) in each group. The mean SDML is not significantly different from the control value in any groups, nor is there any difference between those patients with and without neuropathy and with and without abnormal jitter. In the patients with neuropathy, however, the mean FMNCV is significantly less than in the control group and the patients without neuropathy. In addition the mean area (MCAP) is significantly smaller in the subjects with neuropathy than in the control subjects, and smaller (though not to a significant level) than the patients without neuropathy. Table 3 shows the MUP parameters in the various subject groups. In the patients with neuropathy the motor unit numbers (MUN) are markedly reduced. The mean MUP amplitudes and areas are significantly increased in this group compared with control values and patients without neuropathy. There is a significant increase in the MUP duration in the group with abnormal jitter compared with that with normal jitter. Only the subgroup with abnormal jitter in the group without neuropathy had a mean MUP latency significantly different from the control values. The mean MUP amplitude and area are closely correlated $(\mathrm{r}=0.90 \mathrm{p}<0.001)$ although we have argued previously ${ }^{16}$ that the area is the most reliable index. Both are significantly increased compared with the control subjects in all the patient groups apart from those without neuropathy who had normal jitter. The greatest amplitude and area were obtained in those patients with a neuropathy, and these were significantly larger than in patients without neuropathy.

\section{Discussion}

The results have confirmed the previous findings $^{12}$ that SDML and FMNCV are normal in the multiple sclerosis patients without a clinical peripheral neuropathy. In those who do have evidence of neuropathy there is a significant reduction in FMNCV, MUN and an increase in MUP area and amplitude. Slowing of conduction of this order indicates loss of function in fastest conducting axons which is confirmed by the low MUN. The increased MUP areas and amplitudes can be explained by collateral reinnervation of denervated muscle fibres by the remaining viable motor axons. These findings agree with our previous studies of axonal peripheral neuropathies by these techniques. ${ }^{24-26}$ In the group of patients without a neuropathy, those with normal jitter show no abnormalities of any motor unit parameter. Those with abnormal jitter have significant abnormalities of many motor unit parameters. Each parameter is discussed separately below.

\section{MUP latency}

The mean MUP latency of the abnormal jitter group is significantly prolonged. This latency is the time taken for the action potential to pass from the point of stimulation to the branching of the axon, the time for conduction through the intramuscular nerve branches and for neuro muscular delay. The normal FMNCV suggests that the fastest axons proximal to the intra muscular nerve branches are functioning normally and our attention is therefore drawn to the branches themselves and to neuromuscular trans mission. Increased SFEMG jitter is usuall attributable to the variability of the rise time te्ఠ. threshold of the endplate potential (EPP) when $\overrightarrow{0}$ the safety factor for transmission is reduced. Theoretically, fluctuation in saltatory conduction in the intramuscular branches could produce the same effects on the jitter. Slowing of impulse propagation in immature collateral sprouts involved in reinnervation has been reported ${ }^{21} 22$ and could explain both the abnormal jitter and increased MUP latency. The histological evidence of internodal demyelination in the sural nerve ${ }^{1}$ and the abnormal electrophysiology of motor and sensory axons ${ }^{2} 3$ both suggest dysfunction in the nerve fibre. Increased curare sensitivity has been shown in multiple sclerosis ${ }^{23}$ but no conclusion as to a pre-junctional or post-junctional defect could be made. We are at present investigating neuromuscular transmission in multiple sclerosis and the results will be presented in a subsequent paper.

Unlike the mean MUP latencies the mean SDMLs are not significantly longer than the control values. The SDML is a measure of the fastest conducting axons whilst the mean motor unit potential latencies relate to a wider spectrum of motor axon conduction velocities and sizes. In 
each patient more than two-thirds of the SFEMG jitter values were normal while less than a third were abnormal. This suggests that the lesions are not homogeneous throughout the muscle. A heterogeneous defect would be represented better by the mean MUP latency than by the SDML.

\section{MUP duration}

The MUP duration recorded from a surface electrode includes the differences in conduction time through the intramuscular nerve branches, the neuromuscular transmission delay, the time for propagation of the muscle fibre action potential and in the spatial distribution of the endplates. In this study the mean MUP durations were all greater than the control values but not to the level of significance in most subgroups. However, the MUP durations of the abnormal jitter group were significantly longer than those of the normal jitter group. The MUP duration is the sum of the differences in all the above factors and these cannot be individually distinguished by this technique. However, we have shown an increased MUP duration and prolonged MUP latency of this order in several conditions such as the Guillain-Barre syndrome, ${ }^{24}$ diabetic neuropathy ${ }^{25}$ and myotonic dystrophy. ${ }^{26}$ In these we have concluded that this was due to increased conduction time in the intramuscular nerve branches.

\section{MUP area and amplitude}

The mean MUP area and amplitude are significantly increased in the group with abnormal jitter. This increase could be due to an increased number of muscle fibres within the motor unit or to an increase in the size of the individual muscle fibres. There are no reports of muscle fibre hypertrophy in multiple sclerosis. The increase can therefore be explained by the presence of increased number of muscle fibres in each motor unit by a reinnervation process. It follows that other motor units must have lost the muscle fibres initially. Table 3 shows that there is no significant difference from control values in the number of motor units in any group other than that with neuropathy. Fig 1, however, demonstrates the relationship between MUN and MUP area in the patients (without neuropathy) who had abnormal and normal jitter.

There is a significant negative correlation $(p=<0.02)$ between MUN and MUP area. In addition, although the mean MUN of both control and patient groups lies above the $95 \%$ confidence limit at 100 units all individual figures below that are from patients with abnormal

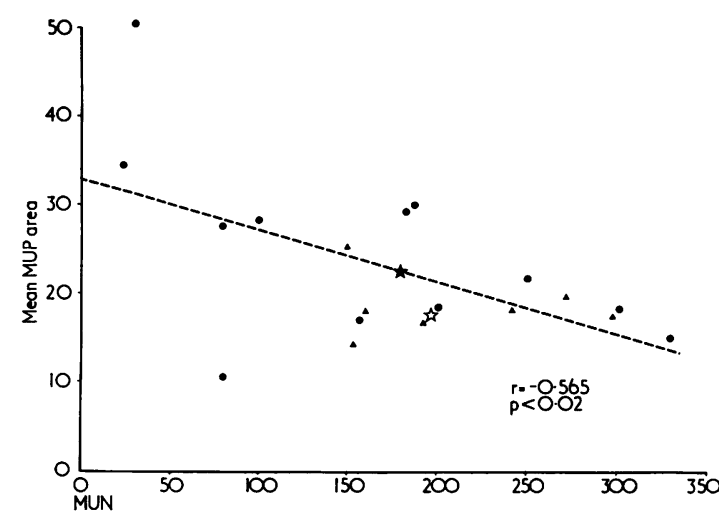

Fig 1 Graph of the relationship between motor unit numbers (MUN) and mean MUP area.

= abnormal jitter

$\Delta=$ normal jitter

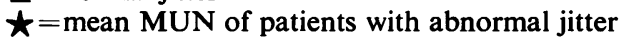
$\hat{\aleph}=$ mean MUN of patients with normal jitter.

jitter. The negative correlation suggests that as the motor unit numbers fall, denervated muscle fibres are reinnervated from intact axons to produce the increase in MUP area.

\section{Conclusion}

Two independent electrophysiological techniques (SFEMG and quantitative electrophysiology) have demonstrated a peripheral nervous system defect in multiple sclerosis. Both have separated a group of subjects with abnormal findings from those with normal findings. The evidence suggests a patchy denervation/reinnervation process due to pathology in the intramuscular nerve network or at the endplate in multiple sclerosis. It is interesting to speculate whether the spectrum of dysfunction in the peripheral nervous system is wide enough to include the three cases of overt clinical neuropathy.

We acknowledge gratefully the advice and encouragement of Professor JA Simpson. We are grateful to the consultant staff of the Institute of Neurological Sciences for referring their patients and to Mrs M McColl for secretarial services. Dr Weir is a Research Fellow supported by the Muscular Dystrophy Group of Great Britain.

\section{References}

1 Pollock M, Calder C, Allpress S. Peripheral nerve abnormality in multiple sclerosis. $A n n$ Neurol 1977; 2:41-8. 
2 Hopf HC. Leitgeschwindigkeit motorischer Nerven bei der multiplen Sklerose und unter dem Einfluss hoher Cortison medikation. Dtsch $Z$ Nervenheilk 1965; 187:522-6.

3 Hopf HC, Eysholdt M. Impaired refractory periods of peripheral nerves in multiple sclerosis. Ann Neurol 1978; 4:499-501.

4 Weir AI, Hansen S, Ballantyne JP. The single fibre electromyographic jitter in multiple sclerosis. $J$ Neurol Neurosurg Psychiatry 1979; 42:1146-50.

5 Adams RD, Kubik CS. The morbid anatomy of the demyelinative diseases. Am J Med 1952; 12: $510-46$.

6 Hasson J. Terry RD, Zimmerman HM. Peripheral neuropathy in multiple sclerosis. Neurology (Minneap) 1958; 8:503-10.

7 Denkler F. Zur kasuistic der multiplen Herdsklerose des Gehirns und Ruckenmarks. Dtsch $Z$ Nervenheilk 1904; 26:233-47.

8 Schob F. Ein Beitrag zur Pathologischen Anatomie der multiplen Sklerose. Mschr Psychiat Neurol 1907; 22:62-87.

9 Schob F. Wurzefibromatose bei multiplen Sklerose. Munch Med Wochenshrift 1912; 2:1831.

10 Schoene WC, Carpenter S, Behan PO, Geschwind N. Onion bulb formations in the central and peripheral nervous system in association with multiple sclerosis and hypertrophic neuropathy. Brain 1977; 100:755-73.

11 Gilliat RW, Goodman HV, Willison RG. The recording of lateral popliteal nerve action potentials in man. J Neurol Neurosurg Psychiatry 1961; 24:305-18.

12 Taraschi G, Lanzi G. Motor nerve conduction velocity in multiple sclerosis. Electroenceph Clin Neurophysiol 1962; 22:54-5.

13 Ginzburg $M$, Lee $M$, Ginsburg J. Correlation between muscle strength and peripheral nerve conduction in multiple sclerosis. Electromyography (Lourain) 1971; 11:491-513.

14 Schumacher GA, Beebe G, Kibler RF et al. Problems of experimental trials of therapy in multiple sclerosis. Ann NY Acad Sci 1965; 122:522-68.

15 Sanders DB, Howard JF, Johns TR. Single fibre electromyography in myasthenia gravis. Neurolgy (Minneap) 1979; 29:68-76.

16 Ballantyne JP, Hansen S. Computer method for the analysis of evoked motor unit potentials. 1 Control subjects and patients with myasthenia gravis. J Neurol Neurosurg Psychiatry 1974; 37:907-15.

17 Ballantyne JP, Hansen S. A new method for the estimation of the number of motor units in a muscle. 1 Control subjects and patients with myasthenia gravis. J Neurol Neurosurg Psychiatry 1974; 37:1187-94.

18 Lenman JAR, Ritchie AE. Clinical Electromyography, 2nd ed. Bath: Pitman, 1977: 60-72.

19 Stalberg E, Ekstedt J, Broman A. The electromyographic jitter in normal human muscles. Electroenceph Clin Neurophysiol 1971; 31: 429-38.

20 Stålberg E, Trontelj JV. Single Fibre Electromyography. Woking: The Mirvelle Press, 1979: 53.

21 Desmedt JE, Borenstein S. Regeneration in Duchenne muscular dystrophy. Arch Neurol 1976; 33:642-50.

22 Stålberg E. Electrogenesis in dystrophic human muscle. In: Rowland, LP, ed. Pathogenesis of human muscular dystrophies. Amsterdam. Exerpta Medica, 1977.

23 Eisen A, Yufe R, Trop D, Campbell I. Reduced neuromuscular transmission safety factory in multiple sclerosis. Neurology (Minneap) 1978; 28:598-602.

24 Martinez-Figueroa A, Hansen S, Ballantyne JP A quantitative electrophysiological study of acute idiopathic polyneuritis. J Neurol Neurosurg Psychiatry 1977; 40:156-61.

25 Hansen S, Ballantyne JP. Axonal dysfunction in the neuropathy of diabetes mellitus: A quantitative electrophysiological study. I Neurol Neurosurg Psychiatry 1977; 40:555-64.

26 Ballantyne JP, Hansen S. New method for the estimation of the number of motor units in a muscle. 2 Duchenne, limb-girdle and facioscapulo humeral, and myotonic muscular dystrophies. J Neurol Neurosurg Psychiatry 1974; 37:1195-201. 